Communications in Physics, Vol. 28, No. 4 (2018), pp. 351-360

DOI:10.15625/0868-3166/28/4/13194

\title{
ADDITIVE EFFECTS OF MACROMOLECULAR CROWDING AND CONFINEMENT ON PROTEIN STABILITY
}

\author{
PHUONG THUY BUI ${ }^{1, \dagger}$ AND TRINH XUAN HOANG ${ }^{2,3}$ \\ ${ }^{1}$ Duy Tan University, \\ 254 Nguyen Van Linh, Thanh Khe, Da Nang, Viet Nam \\ ${ }^{2}$ Institute of Physics, \\ Vietnam Academy of Science and Technology, \\ 10 Dao Tan, Ba Dinh, Ha Noi, Viet Nam \\ ${ }^{3}$ Graduate University of Science and Technology, \\ Vietnam Academy of Science and Technology, \\ 18 Hoang Quoc Viet, Cau Giay, Ha Noi, Viet Nam \\ ${ }^{\dagger}$ E-mail: buiphuongthuyphys@gmail.com
}

Received 16 October 2018

Accepted for publication 30 November 2018

Published 15 December 2018

\begin{abstract}
Folding of protein in vivo typically occurs in a solution highly crowded by macromolecules and in a confined space. It has been found that the effects of macromolecular crowding and confinement are similar in terms of the enhancement of protein stability. However, these effects are often considered separately in theoretical and simulation studies. In this study, by using coarse-grained models and Langevin dynamics, we show that the two effects are additive to each other when they are both present. Both crowding and confinement give rise to the folding temperature and the folding stability of protein. It is shown that the folding free energy change due to crowding in the confined condition can be fitted to Minton scaled particle theory by assuming a linear dependence of the effective radius of the protein unfolded state on the volume fraction of crowders.
\end{abstract}

Keywords: confinement, crowding, molecular dynamics, protein folding.

Classification numbers: 87.15.M-; 87.64.K-. 


\section{INTRODUCTION}

The cytosol of a living cell is crowded by a large number of macromolecules including many proteins, ribonucleic acids (RNAs) and their soluble complexes, which occupy up to 40\% of the cytosolic volume. Furthermore, subcellular organelles create confining environments that influence the dynamics of functional molecules. These crowded and confined conditions yield significant impacts on the equilibria and rates of a variety of macromolecular reactions [1,2]. Particularly, both crowding and confinement have been shown to increase the stability and the folding rate of proteins. Certain types of cellular confinement, such as that of the ribosomal exit tunnel $[3,4]$ and the cage of chaperonin $[5,6]$, are believed to functionally improve the folding efficiency of proteins. Due to the necessity of identifying the effect for a particular type of condition, crowding and confinement are typically considered separately in theoretical and experimental studies. Thus, it is not clear whether these effects add up or annihilate each other in conditions where they coexist. In this paper, we will focus on studying the combined effect of macromolecular crowding and confinement on protein stability. For the latter we are concerned with both the thermal stability and the folding stability of protein. The thermal stability is associated with the folding transition temperature (or folding temperature), whereas the folding stability corresponds to the folding free energy, which is the free energy difference between the folded state and the unfolded state of a protein.

The primary effect of both macromolecular crowding and confinement on protein folding is excluded volume. In the case of crowding, the protein is enclosed in a floppy pocket of available space left by the crowders, which fluctuates continuously due to the motions of crowders. In the case of confinement, fixed boundaries rule out protein conformations which stick out from the confined volume. In both cases, the volume exclusion would be adversely 'felt' by the unfolded state more than the folded state due to differences in their effective sizes and their conformational entropies, leading to an increase of the folded state's stability. The strength of this excluded volume effect has been assessed in many studies by theory, simulation and experiment.

The scaled particle theory (SPT) [7] allows one to obtain the free energy of inserting a hard sphere of a given size in a hard sphere fluid. Minton [8] used the SPT to calculate the folding free energy change as the difference between the insertion free energies for the folded and unfolded states by assuming that both states are effective hard spheres. Zhou [9] calculated the free energy for the folded state by using the SPT but obtained the free energy for the unfolded state through a model which treats the unfolded state as a self-avoiding walk in presence of spherical crowders. These theories, as well as a number of other SPT-based theories, predicted an increase of a few $k_{B} T$ of the folding stability in crowded conditions. The free energy change and its dependence on the crowder volume fraction, however, depend on the theory. Mittal and Best [10] recently reported simulation results which mostly agree with Minton's theory and suggested that the effective radius of the unfolded state should be about 0.8 times its radius of gyration. By using theoretical models with a Gaussian chain and also a self-avoiding walk under spherical confinement, Zhou showed that strong confinement can stabilize protein by over $10 k_{B} T$ [9]. All-atom simulations carried out by Pande's group showed that confinement alone stabilizes the folded state but confining both protein and solvent may lead to solvent-mediated effect that destabilizes the folded state [11].

Experimentally, the stabilizing effect of inert excluded volume crowders on protein's folded state has been confirmed with a stability increase of 1-2 $k_{B} T$ by using synthetic polymer crowders 


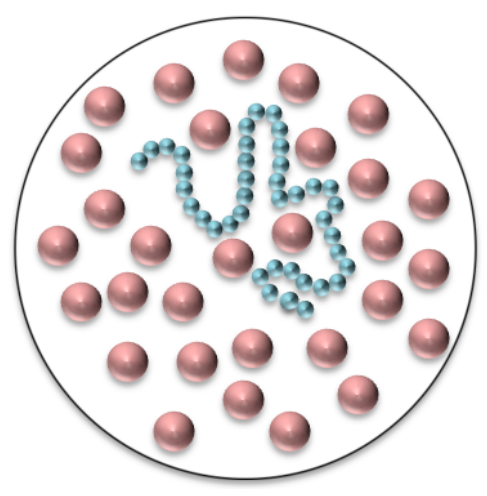

Fig. 1. Sketch of the models of a protein and crowders confined in a spherical container.

such as Ficall [12], Dextran [13] and PEG [14]. Destabilizing effects, however, are observed for two protein crowders, lyzozyme and bovine serium albumin (BSA), on the folding stability of chymotrypsin inhibitor 2 (CI2) [15], suggesting that the interaction between the crowder and the protein may enthalpically favor the protein unfolded state [16]. Confinements created by nanopores in gel [17] and in reverse micelle [18] have been reported to increase the protein stability and folding efficiency.

In the present work, we study the combined effect of macromolecular crowding and confinement on the folding stability by using molecular dynamics method with coarse-grained models. Our consideration is limited to spherical crowder and spherical confinement. We also check if the free energy change obtained from the simulations can be fitted to Minton's scaled particle theory.

\section{MODELS AND METHODS}

\section{II.1. Models and simulation method}

We consider the same Go-like model [19] for protein as described in Ref. [3]. In this model, amino acids are represented by spherical beads centered on the positions of the $\mathrm{C}_{\alpha}$ atoms. The beads forming a native contact, i.e. a contact present in the protein's native state, interact via the 6-12 Lennard-Jones (LJ) potential with the energy $\epsilon$ being the depth of the potential. On the other hand, the beads that do not form a native contact interact via a short-ranged repulsive potential. The model also includes local potentials for the bond angles and dihedral angles which are centered on native values of these angles.

The crowders are modeled as soft spheres of radius $R_{c}=10 \AA$. Consider a system of one protein and $M$ crowders confined in a spherical container of radius $R_{\text {wall }}$ (Fig. 1). The interactions between any two crowders, between an amino acid and a crowder, between an amino acid and the container wall, and between a crowder and the container wall are all repulsive at short distances and are given by the truncated and shifted LJ potential

$$
V_{\text {rep }}(r)= \begin{cases}4 \epsilon\left[(\sigma / r)^{12}-(\sigma / r)^{6}\right]+\epsilon & , r \leq 2^{1 / 6} \sigma \\ 0 & , r>2^{1 / 6} \sigma\end{cases}
$$


where $\sigma=5 \AA$ and $r$ is the distance between an amino acid and the nearest virtual residue or between the two nearest virtual residues. The virtual residues are considered to be of the same size as amino acid and embedded under the surface of a crowder or the container wall (see Ref. [4] for an illustrated definition of virtual residue). For comparison, we consider also the case of a crowded condition but without confinement. For this case, the protein and crowders are enclosed in a cubic box of a side length $L=100 \AA$ with periodic boundary conditions for the crowders. The crowder volume fraction is given by $\phi_{c}=M(4 \pi / 3) R_{c}^{3} / V$, where $M$ is the number of crowders and $V$ is the volume of container or the simulation box.

The motions of the protein and the crowders follow the Langevin equation. We assume that all amino acids have the same mass, $m$, while the crowder has the mass $m_{c}=m\left(R_{c} / R_{a}\right)^{3}$, where $R_{a}=2.5 \AA$ is the Van der Waals radius of amino acid. The friction coefficients $\zeta$ for amino acid and $\zeta_{c}$ for crowder are chosen according to the Stokes's law for the friction of spherical object in a viscous fluid, i.e. $\zeta_{c}=\zeta\left(R_{c} / R_{a}\right)$. The Langevin equations are integrated by using a Verlet algorithm introduced in Ref. [3] with time step $\Delta t=0.002 \tau$, where $\tau=\sqrt{m \sigma^{2} / \epsilon}$ is the time unit in the system. In the simulations, we use $\zeta=5 m \tau^{-1}$, for which the dynamics can be considered as overdamped [20,21].

For studying the folding properties, we consider the specific heat and the free energy of the protein. The specific heat depends on temperature and is defined as

$$
C(T)=\frac{\left\langle E^{2}\right\rangle-\langle E\rangle^{2}}{k_{B} T^{2}},
$$

where $k_{B}$ is the Boltzmann constant, $T$ is the absolute temperature in units of $\epsilon / k_{B}$, and $E$ is the energy of the protein (equal to the sum of the potential and kinetic energies of the protein). The free energy of protein at a given temperature $T$ is considered as a function of the fraction of native contacts $Q$ and is defined as

$$
F(Q)=-k_{B} T \ln P(Q),
$$

where $Q=N_{c} / N_{c}^{*}$ is the ratio of the number of native contacts of a given conformation $\left(N_{c}\right)$ to that of the native state $\left(N_{c}^{*}\right), P(Q)$ is the probability of observing conformations having the fraction of native contacts $Q$. Here, $Q$ represents a folding reaction coordinate $(0 \leq Q \leq 1)$ with $Q=0$ corresponding to a completely unfolded conformation and $Q=1$ corresponding to the native state. The averages of $E$ and $P(Q)$ are calculated from the equilibrium simulation data at various temperatures with the use of the weighted histogram method [22].

The folding free energy is the free energy difference between the folded state $(N)$ and the unfolded state $(U)$ and can be calculated from the free energy profile $F(Q)$ as [10]

$$
\Delta F_{N-U} \equiv F_{N}-F_{U}=-k_{B} T \ln \left(\frac{\int_{Q_{\ddagger}}^{1} e^{-\beta F(Q) d Q}}{\int_{\ddagger}^{Q_{\ddagger}} e^{-\beta F(Q) d Q}}\right),
$$

where $Q_{\ddagger}$ corresponds to the location of the transition state (†) along $Q$. The change of folding free energy due to the effect of crowders is given by

$$
\Delta \Delta F_{N-U}=\Delta F_{N-U}\left(\phi_{c}\right)-\Delta F_{N-U}(0),
$$

where $\Delta F_{N-U}\left(\phi_{c}\right)$ is the folding free energy found at the crowder volume fraction $\phi_{c}$.

We calculate also the gyration radius $R_{g}^{U}$ of the unfolded state and the gyration radius $R_{g}^{N}$ of the folded state from the simulations. These radii are defined as averages in the corresponding 
ensembles of unfolded and folded conformations:

$$
\begin{aligned}
R_{g}^{U} & =\frac{\int_{0}^{Q_{\ddagger}} \bar{R}_{g}(Q) e^{-\beta F(Q)} d Q}{\int_{0}^{Q_{\ddagger}} e^{-\beta F(Q)} d Q}, \\
R_{g}^{N} & =\frac{\int_{Q_{\ddagger}}^{1} \bar{R}_{g}(Q) e^{-\beta F(Q)} d Q}{\int_{Q_{\ddagger}}^{1} e^{-\beta F(Q)} d Q},
\end{aligned}
$$

where $\bar{R}_{g}(Q)$ is the mean radius of gyration of conformations having the fraction of native contacts $Q$.

\section{II.2. Minton scaled particle theory}

The scaled particle theory of hard particle mixture fluid [23] gives an estimate of the Helmholtz free energy change of inserting a hard sphere particle of radius $R$ in a fluid of hard sphere crowders of radius $R_{c}$ as given by $[8,10]$

$$
\frac{\Delta F}{k_{B} T}=-\ln \left(1-\phi_{c}\right)+\rho y\left(3+3 y+y^{2}\right)+\rho^{2} y^{2}(9 / 2+3 y)+3 \rho^{3} y^{3},
$$

where $\phi_{c}$ is the volume fraction of crowders, $\rho=\phi_{c} /\left(1-\phi_{c}\right)$, and $y=R / R_{c}$. Minton [8] treated the folded state and unfolded state as effective hard spheres. Assume that these spheres have the radii $a_{N}$ and $a_{U}$, respectively. The folding free energy change in Eq. (5) can be calculated as

$$
\begin{aligned}
\Delta \Delta F_{N-U} & =\Delta F_{N-U}\left(\phi_{c}\right)-\Delta F_{N-U}(0) \\
& =F_{N}\left(\phi_{c}\right)-F_{U}\left(\phi_{c}\right)-F_{N}(0)+F_{U}(0) \\
& =\left[F_{N}\left(\phi_{c}\right)-F_{N}(0)\right]-\left[F_{U}\left(\phi_{c}\right)-F_{U}(0)\right] \\
& =\Delta F_{N}-\Delta F_{U},
\end{aligned}
$$

where $\Delta F_{N}$ and $\Delta F_{U}$ are obtained from Eq. (8) by putting $R$ equal to $a_{N}$ and $a_{U}$, respectively. In our application of the scaled particle theory, $a_{N}$ is chosen to be equal to the radius of gyration of the native conformation, whereas $a_{U}$ is a parameter for fitting with simulation data.

\section{RESULTS AND DISCUSSION}

For studying the effects of crowding and confinement on protein folding we consider two small globular proteins, the B1 domain of protein G (GB1) with the protein data bank (PDB) code 1PGA and the Z domain of Staphylococcal protein (SpA) with PDB code 2SPZ. The GB1 has a length of $N=56$ amino acids whereas the SpA has the length of $N=58$. For protein GB1, we carried out the simulations of the protein in various crowded conditions of the crowder volume fraction $\phi_{c}$ varied between 0 and 0.3 , for the periodic boundary condition (pdb) and two cases of spherical confinement with $R_{\text {wall }}=100 \AA$ and $R_{\text {wall }}=50 \AA$. For protein SpA, only the crowding and the confinement with $R_{\text {wall }}=100 \AA$ are considered.

The specific heat is a fundamental thermodynamic function indicative of a phase transition. In protein folding, the temperature of the peak of the specific heat as a function of temperature corresponds to the folding transition temperature or folding temperature, $T_{f}$, whereas the height of the peak, $C_{\max }$, signifies the degree of cooperativity of the folding transition [24]. Figure 2(a) shows the dependence of the specific heat on temperature for protein GB1 obtained at different values of crowder volume fraction under the confinement of $R_{\text {wall }}=50 \AA$. It is shown that as 

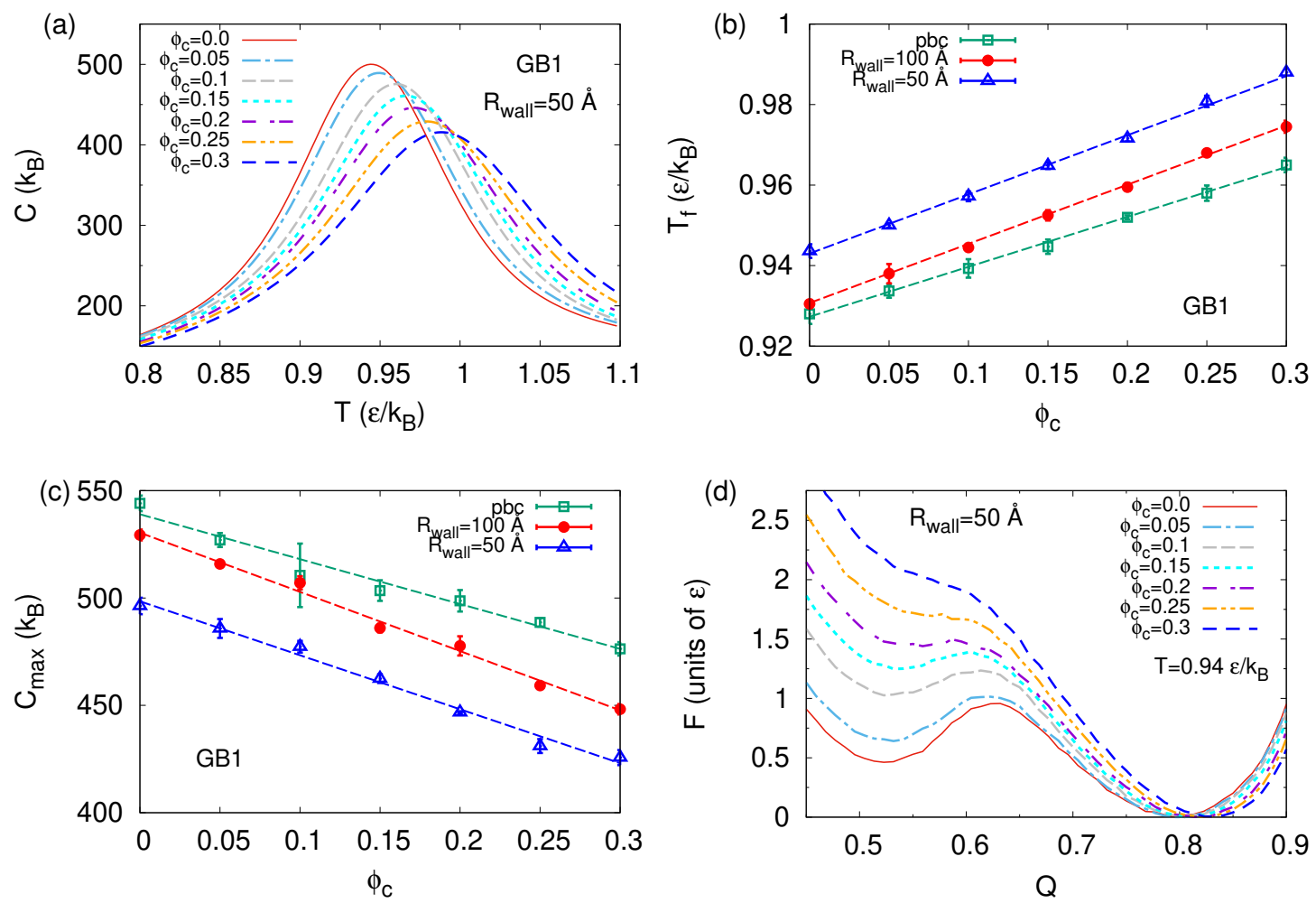

Fig. 2. (a) Comparison of the specific heats of the protein GB1 (PDB code: 1PGA) for different crowder volume fractions $\phi_{c}$ (as indicated) under spherical confinement with $R_{\text {wall }}=50 \AA$. (b, c) Dependence of the maximum of the specific heat $C_{\max }$ (c) and the folding temperature $T_{f}(\mathrm{~d})$ on $\phi_{c}$ for three conditions: with periodic boundary condition (pbc) (squares), with spherical confinements of $R_{\text {wall }}=100 \AA$ (circles) and $50 \AA$ (triangles). (d) Dependence of the free energy, $F$, on the fraction of native contacts, $Q$, at temperature $T=0.94 \epsilon / k_{B}$ for GB1 for different values of $\phi_{c}$ and with $R_{\text {wall }}=50 \AA$.

$\phi_{c}$ increases, the temperature of the specific heat peak also increases but the height of the peak decreases. Thus, under crowded condition the protein is thermally more stable with an increased folding temperature $T_{f}$ but the folding transition is less cooperative than in condition without crowders. Figures 2(b) and 2(c) show that $T_{f}$ increases linearly and $C_{\max }$ decreases linearly with $\phi_{c}$ for all three types of boundary conditions, i.e. pbc or with confinements. Note that as the confinement degree is gradually enforced, from no confinement (pbc) to the confinements of $R_{\text {wall }}=100 \AA$ and then $R_{\text {wall }}=50 \AA, T_{f}$ also increases and $C_{\text {max }}$ decreases. Thus, the effects of crowding and confinement add up to each other, both in terms of thermal stability and folding cooperativity of protein. The slope of the linear dependence however slightly increases as one moves from the pbc to the confined conditions, indicating some small enhancing effect of confinement on the effect of crowding. 
Figure 2(d) shows the dependence of the free energy on the fraction of native contacts $Q$ at a temperature slightly lower than $T_{f}$ for the crowded and confined conditions as in Fig. 2(a). It is shown that for $\phi_{c} \leq 0.2$ the free energy profile has two minima corresponding to the equilibrium of the folded (at higher $Q$ ) and unfolded (at lower $Q$ ) states. These states are separated by a barrier (the position of the maximum of the barrier corresponds to the transition state). At higher $\phi_{c}$ the minimum corresponding to the unfolded state disappears. As $\phi_{c}$ increases the free energy difference between the unfolded state and the folded state also increases, meaning that the absolute value of the folding free energy also increases. Thus, it is shown that due to crowding the folded state becomes thermodynamically more stable than the unfolded state. This result is in agreement with many previous theoretical and simulation studies on the effect of crowding $[8,10]$, even though our result was obtained with the combined crowded and confined conditions.

In Fig. 3(a), we show the dependence of the folding free energy change, $\Delta \Delta F_{N-U}$, on $\phi_{c}$ for protein GB1 with three cases of boundary conditions, together with the fits of the simulation data to the scaled particle theory. Here, all the free energy changes are shown with a reference to the folding free energy of the protein in the unconfined condition without crowder, i.e. the pbc case with $\phi_{c}=0$. Note that $\Delta \Delta F_{N-U}$ decreases either when $\phi_{c}$ increases or when one moves from the pbc condition (unconfined) to the confined boundary conditions of decreasing radius $R_{\text {wall }}$. This result indicates that the effects of crowding and confinement are also additive to each other in terms of the folding free energy change. Fig. 3(a) also shows that the scaled particle theory with Minton's approximation of effective hard spheres can be fitted quite well to the simulation results given that the effective radius of the unfolded state, $a_{U}$, depends linearly on $\phi_{c}$. Fig. 3(b) shows that both $a_{U}$ and the radius of gyration of the unfolded state, $R_{g}^{U}$ decrease linearly with $\phi_{c}$ but $a_{U}$ decreases faster than $R_{g}^{U}$. Furthermore, $a_{U}$ is always smaller than $R_{g}^{U}$. Fig. 3(b) also shows that the radius of gyration of the folded state, $R_{g}^{N}$, is almost independent of $\phi_{c}$. Fig. 3(c) shows that the ratio $a_{U} / R_{g}^{U}$ strongly depends on the boundary condition and also decreases with $\phi_{c}$. For the periodic boundary condition, the ratio $a_{U} / R_{g}^{U}$ is about 0.8 and does not change significantly with $\phi_{c}$, in agreement with previous result of Mittal and Best [10]. For the two confined conditions, we find that this ratio is larger than 0.8 and decreases with $\phi_{c}$. In particular, for $R_{\text {wall }}=100 \AA$, $a_{U} / R_{g}^{U} \approx 0.9$ at $\phi_{c}=0.05$ and $\approx 0.83$ at $\phi_{c}=0.3$. The corresponding values of this ratio are 0.93 at $\phi_{c}=0.05$ and 0.86 at $\phi_{c}=0.3$ for $R_{\text {wall }}=50 \AA$. Thus, under confinement, the effective hard sphere radius of the unfolded state, as being considered in Minton theory, is closer to the radius of gyration of this state than in unconfined condition. In other words, the hard sphere approximation is better founded under confinement.

Figure 4 shows the dependence of $\Delta \Delta F_{N-U}$ on $\phi_{c}$ for protein SpA under confinement with $R_{\text {wall }}=100 \AA$ and the fit to the scaled particle theory. The results are similar to the case of GB1, except that the dependence of $a_{U}$ and $R_{g}^{U}$ on $\phi_{c}$ is weaker than for GB1 (Fig. 4(b)). For SpA, the ration $a_{U} / R_{g}^{U}$ is close to 0.82 and almost does not depend on $\phi_{c}$ (Fig. 4(c)).

Our results were obtained for small single-domain proteins which are two-state folders. It can be expected that the properties found also apply for larger two-state proteins. For much longer proteins, the native state typically is multi-domain; folding is autonomous for each domain but the domains may also influence each other. In that case, the stabilizing effects of crowder and confinement on the protein are still present but they can be quantitatively more complicated than for small proteins. 
(a)

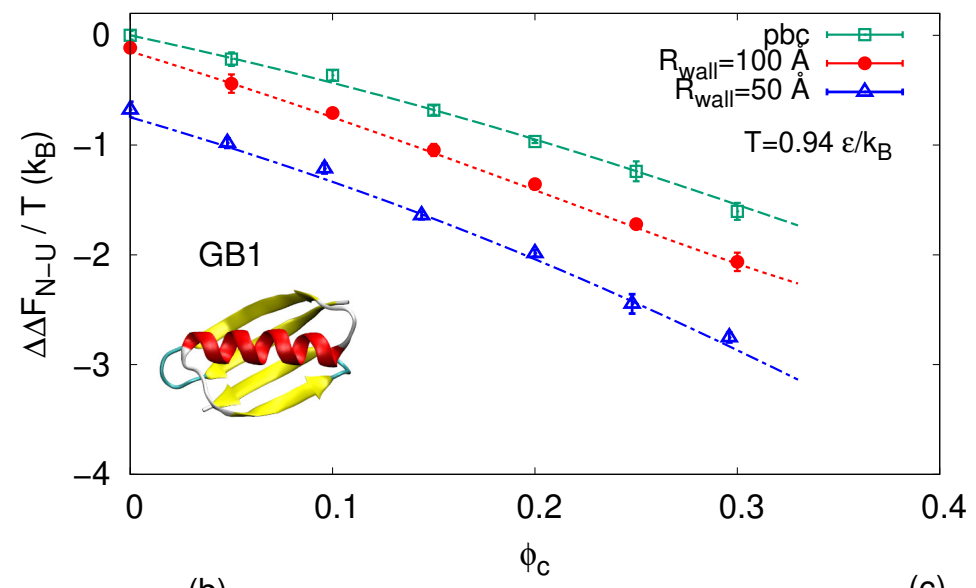

(b)

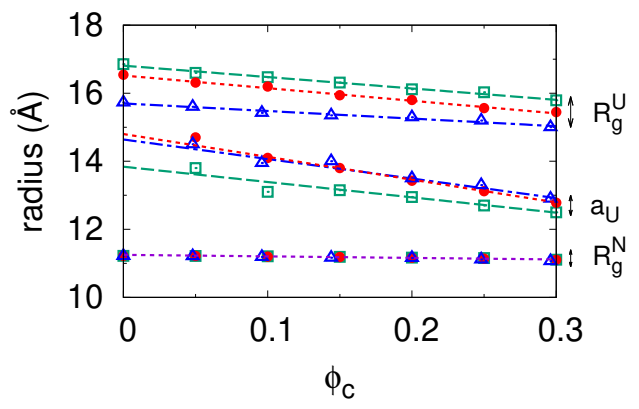

(c)

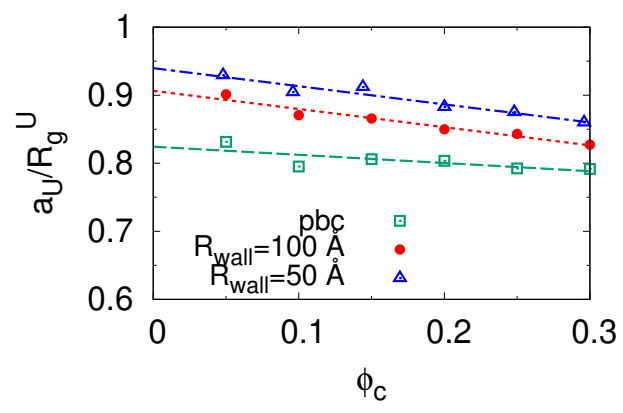

Fig. 3. (a) Dependence of the folding free energy change on the volume fraction of crowders obtained at $T=0.94 \epsilon / k_{B}$ for protein GB1 three cases of boundary conditions: periodic boundary condition (pbc) (squares), spherical confinement with $R_{\text {wall }}=100 \AA$ (circles) and spherical confinement with $R_{\text {wall }}=50 \AA$ (triangles). The data points obtained from the simulations are fitted to the scaled particle theory (lines) with an assumption that $a_{U}$ depends linearly on $\phi_{c}$. The native conformation of the protein is shown as inset. $(\mathrm{b}, \mathrm{c})$ Dependence of the effective radius of the unfolded state, $a_{U}$, and the radii of gyration, $R_{g}^{N}$ and $R_{g}^{U}$, of the folded and unfolded states, respectively, (b) and the ratio of $a_{U} / R_{g}^{U}$ on $\phi_{c}$ (c) on the crowder volume fraction $\phi_{c}$ for the three boundary conditions as shown in (a).

The dependence of the effective radius of the protein unfolded state on the volume fraction of crowders can be explained based on consideration of osmotic pressure. The osmotic pressure induced by crowders increases linearly with the crowders volume fraction. One may consider the unfolded state as a spherical balloon with an osmotic pressure inside the balloon induced by the unfolded chain itself. If the osmotic pressure inside the balloon depends linearly on the balloon radius, then it comes out that the effective radius of the unfolded state depends linearly on the crowder volume fraction. The linear dependence of the unfolded chains osmotic pressure on the balloon radius, however, is a property of the unfolded state and may result from both entropic and enthalpic contributions in the unfolded protein. Our simulation result about the linear dependence 
of the unfolded state radius of gyration on crowder volume fraction obtained for two different proteins suggests that this property is not quite accidental.

(a)

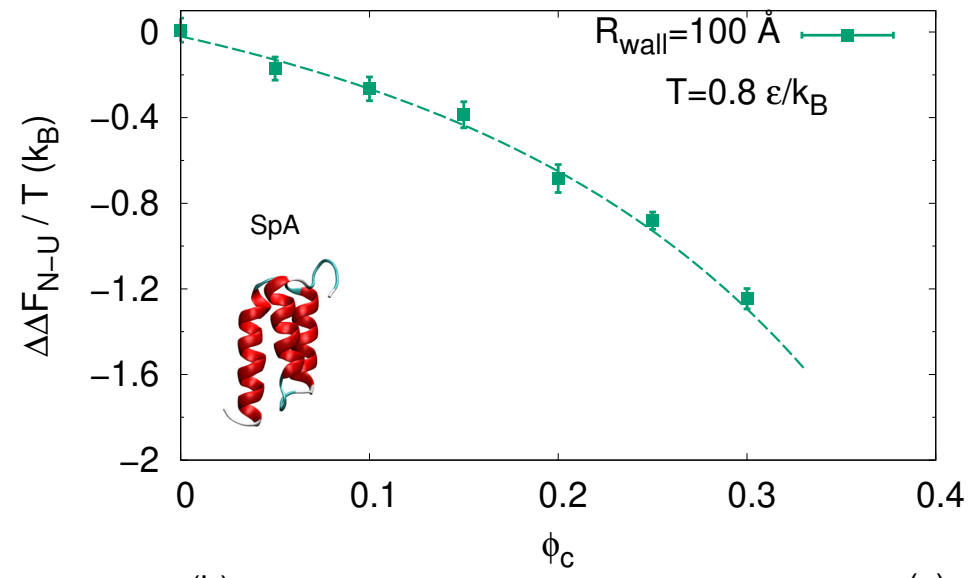

(b)

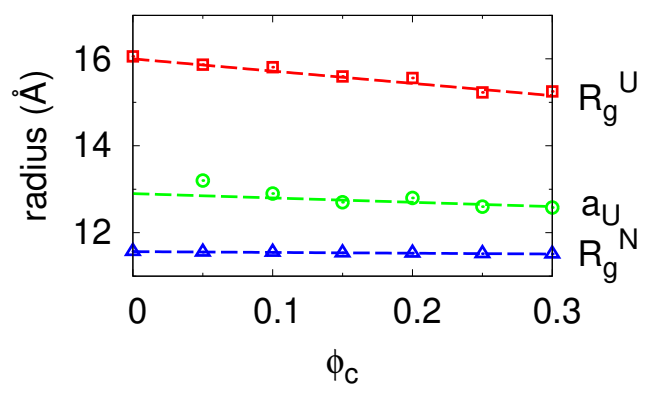

(c)

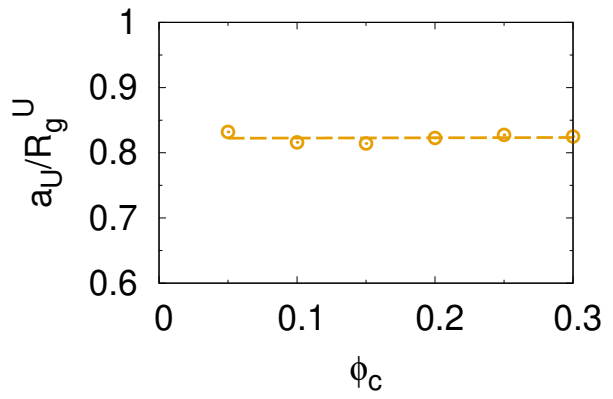

Fig. 4. Same as Fig. 3 but for protein SpA (PDB code: 2SPZ) and only with the spherical confinement of $R_{\text {wall }}=100 \AA$. The folding free energy changes shown in (a) are obtained by simulations at temperature $T=0.8 \epsilon / k_{B}$, which is slightly below the folding temperature of this protein.

\section{CONCLUSIONS}

Macromolecular crowding and confinement are cellular conditions that increase the stability of protein, which is true for both the thermal stability of the native state, associated with the folding temperature, and the folding stability, i.e. the thermodynamic stability of the folded state with respect to the unfolded state. This stability increase is due to the excluded volume effects of crowding and confinement. We have shown that in conditions when both the crowding and confinement are present, these effects are additive to each other giving rises to the folding temperature and the folding free energy of protein approximately in accumulative way. Our simulations show that under crowded and confined conditions, the folding temperature increases linearly with the crowder volume fraction and the height of the specific heat decreases linearly with the latter. 
Also under these conditions, the dependence of folding free energy change on crowder volume fraction can be fitted by Minton scaled particle theory by assuming that the effective size of the unfolded state depends linearly on the crowder volume fraction. Our results about the obtained linear dependences can be useful in the case when one would like to extrapolate experimental or simulation data to predict the folding temperature or the free energy change for a wide range of crowder volume fraction.

\section{ACKNOWLEDGMENT}

We acknowledge the financial support of the International Centre of Physics at the Institute of Physics, Vietnam Academy of Science and Technology.

\section{REFERENCES}

[1] H.-X. Zhou, G. Rivas and A. P. Minton, Annu. Rev. Biophys. 37 (2008) 375.

[2] A. H. Elcock, Curr. Opin. Struct. Bio. 20 (2010) 196.

[3] P. T. Bui and T. X. Hoang, J. Chem. Phys. 144 (2016) 095102.

[4] P. T. Bui and T. X. Hoang, J. Chem. Phys. 149 (2018) 045102.

[5] M. Mayhew, A. C. da Silva, J. Martin, H. Erdjument-Bromage, P. Tempst and F. U. Hartl, Nature 379 (1996) 420.

[6] Y.-C. Tang, H.-C. Chang, A. Roeben, D. Wischnewski, N. Wischnewski, M. J. Kerner, F. U. Hartl and M. HayerHartl, Cell 125 (2006) 903.

[7] J. Lebowitz and J. Rowlinson, J. Chem. Phys. 41 (1964) 133.

[8] A. P. Minton, Biophys. J. 78 (2000) 101.

[9] H.-X. Zhou, Arch. Biochem. Biophys. 469 (2008) 76.

[10] J. Mittal and R. B. Best, Biophys. J. 98 (2010) 315.

[11] D. Lucent, V. Vishal and V. S. Pande, Proc. Natl. Acad. Sci. USA 104 (2007) 10430.

[12] D. S. Spencer, K. Xu, T. M. Logan and H.-X. Zhou, J. Mol. Biol. 351 (2005) 219.

[13] P. McPhie, Y.-s. Ni and A. P. Minton, J. Mol. Biol. 361 (2006) 7.

[14] Y. Sasaki, D. Miyoshi and N. Sugimoto, Nucl. Acid. Res. 35 (2007) 4086.

[15] A. C. Miklos, M. Sarkar, Y. Wang and G. J. Pielak, J. Am. Chem. Soc. 133 (2011) 7116.

[16] H.-X. Zhou, FEBS Lett. 587 (2013) 394.

[17] D. Bolis, A. S. Politou, G. Kelly, A. Pastore and P. A. Temussi, J. Mol. Biol. 336 (2004) 203.

[18] R. W. Peterson, K. Anbalagan, C. Tommos and A. J. Wand, J. Am. Chem. Soc. 126 (2004) 9498.

[19] N. Go, Ann. Rev. Biophys. Bioeng. 12 (1983) 183.

[20] D. Klimov and D. Thirumalai, Phys. Rev. Lett. 79 (1997) 317.

[21] T. X. Hoang and M. Cieplak, J. Chem. Phys. 112 (2000) 6851.

[22] A. M. Ferrenberg and R. H. Swendsen, Phys. Rev. Lett. 63 (1989) 1195.

[23] T. Boublík, Mol. Phys. 27 (1974) 1415.

[24] H. Kaya and H. S. Chan, Prot. Struct. Func. Bio. 40 (2000) 637. 